\title{
An Autonomous Sensor System Architecture for Active Flow and Noise Control Feedback
}

\author{
William M. Humphreys, Jr. ${ }^{*}$ and William G. Culliton ${ }^{\dagger}$ \\ NASA Langley Research Center, Hampton, Virginia 23681
}

\begin{abstract}
Multi-channel sensor fusion represents a powerful technique to simply and efficiently extract information from complex phenomena. While the technique has traditionally been used for military target tracking and situational awareness, a study has been successfully completed that demonstrates that sensor fusion can be applied equally well to aerodynamic applications. A prototype autonomous hardware processor was successfully designed and used to detect in real-time the two-dimensional flow reattachment location generated by a simple separated-flow wind tunnel model. The success of this demonstration illustrates the feasibility of using autonomous sensor processing architectures to enhance flow control feedback signal generation.
\end{abstract}

\section{Nomenclature}

$\begin{array}{lll}\alpha & = & \text { leading edge angle (LEA), deg } \\ C_{p}, & = & \text { RMS pressure coefficient } \\ i & = & \text { array sensor number } \\ f_{O S C} & = & \text { model leading edge oscillation frequency, Hz } \\ T & = & \text { integration time, sec } \\ v_{k} & = & \text { sensor } \mathrm{k}^{\text {th }} \text { voltage sample, volts } \\ \bar{v} & = & \text { sensor mean voltage, volts } \\ V(t) & = & \text { processor input voltage, volts } \\ V_{R M S} & = & \text { root-mean-square voltage, volts } \\ V_{R E F} & = & \text { digital-to-analog converter reference voltage, }=10 \text { volts } \\ V_{O U T} & = & \text { processor output voltage, volts } \\ U_{\infty} & = & \text { freestream velocity, } \mathrm{m} / \mathrm{sec} \\ x_{r} & = & \text { reattachment distance, } \mathrm{mm}\end{array}$

\section{Introduction}

\begin{abstract}
CTIVE flow and noise control research involves the design of open- and closed-loop control circuits capable of manipulating physical flow phenomena with the intent of maintaining the phenomena at a desired state. The research has an extensive history dating back many decades, and excellent reviews of the subject are available in the literature. ${ }^{1-4}$ Numerous computational and experimental studies have focused on open-loop control to understand the underlying physics of the phenomena and to permit the design of proper flow actuators. More recently, emphasis has been placed on the development of closed-loop control. This is a more challenging task because the control system must be robust in its handling of unknown uncertainties in the flow state. Practically, closed-loop controllers require the efficient design of not only actuators but also sensors and sensor data processing systems. Figure 1 depicts a typical closed-
\end{abstract}

\footnotetext{
* Senior Research Scientist, Aeroacoustics Branch, Senior Member AIAA.

${ }^{\dagger}$ Research Technician, Advanced Sensing and Optical Measurement Branch.
} 
loop controller, where one or more actuators interact with a flow system and an array of sensors provide feedback information to the controller. The goal of the sensor array is to measure the real-time state of the flow and quickly pass that information to the controller to allow the actuators to influence the phenomena and maintain a desired state. The efficiency of the sensor array subsystem and the handling of the sensor data influences the ultimate controllability of the system.

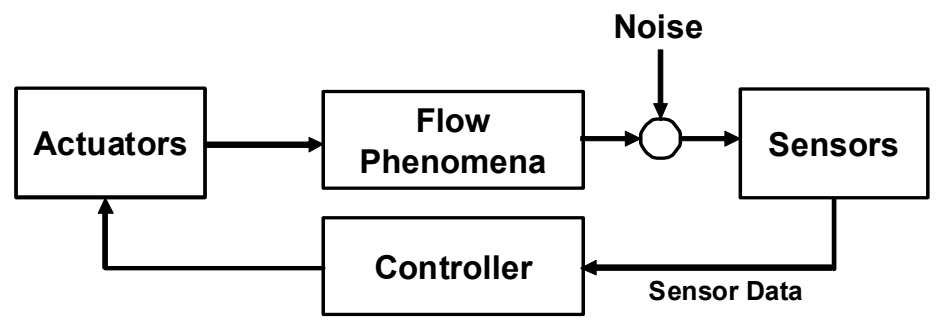

Figure 1. Typical Closed-Loop Control System.

Significant research has been conducted in the development of flow actuators and their use has been documented in numerous applications including circulation, shear layer, and jet noise control. ${ }^{5-6}$ Concurrent with the actuator development has been the creation of smaller, more efficient sensors that can be embedded in the skin of vehicles to provide measurement of the flow state. ${ }^{7}$ To maximize the sensor observability (i.e., the ability of the sensors to reliably detect the flow state) and thus improve the controllability of the system, large heterogeneous arrays of sensors are often required. However, as the number of array sensors increases, significant barriers to practical deployment of this technology emerge. These barriers fall into three main categories, namely powering of the array sensors, interconnection of the sensor outputs, and processing of the sensor data. Of these three categories, the processing of sensor data is an area where significant progress has been shown over the last two decades. Nonetheless, challenges remain if the goal is to properly use the information collected by hundreds or thousands of sensors in a control system.

To illustrate the challenges faced when deploying very large sensor arrays as part of a control system, consider the classical centralized sensor data acquisition technique shown in Figure 2. In this approach, individual sensor outputs are conditioned in the analog domain and transmitted over multiple data channels

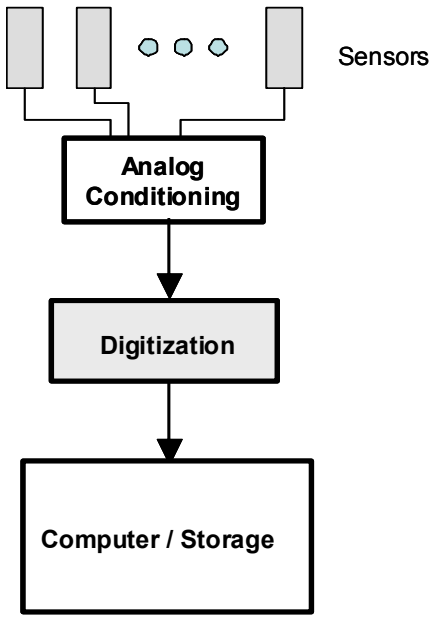

Figure 2. Classical Data Acquisition. to a centralized processor that digitizes the signals and extracts the desired data. The problems with this architecture are two-fold. First, such architectures exhibit poor scalability. As the number of sensors increases, the number and bandwidth of the corresponding communication channels interfacing to the central processor must also increase. Second, there is a large increase in the burden undertaken by the central processor in digitizing and reducing the sensor data as the size of the system increases. The end result is the transmission and processing segments of the system can quickly become overwhelmed at large scale. The situation is not unlike that faced by the early semiconductor industry after the invention of the integrated circuit. As the number of transistors included in an integrated circuit increased from a few hundred to many millions, it became necessary to develop new and advanced signal handling and interconnection topologies. 
To improve the scalability of sensor arrays used for flow and noise control and reduce the burden on the processing hardware, a study was undertaken to devise new architectures to efficiently condition and process the sensor outputs. The ultimate need is for the development of an "integrated sensory agent" composed of the sensors, a de-centralized power distribution network, a reconfigurable signal interconnect (which could be wired or wireless), and a distributed signal conditioning and processing system. The scope of the present study focused on the development of an efficient, scalable processing architecture. The proposed signal conditioning and processing architecture is based on sensor fusion and data mining methods adapted from military target tracking and classification algorithms. ${ }^{8-10}$ This paper describes the design philosophy employed to develop the architecture, and discusses the construction and evaluation of a prototype hardware processor based on the new architecture.

\section{Design Philosophy}

A successful design for processing data from sensor arrays used for flow and noise control must address two issues. First, the processing hardware must be autonomous in nature, operating continuously on time history information derived from the sensors independent of the state of the flow control feedback loop. Second, the processor must generate a stream of relevant information, not simply produce reams of raw data. Given these attributes, sensor fusion methodologies are ideally suited to the task of providing feedback information to flow controllers like that shown in Fig. 1. Sensor fusion is defined as a multilevel process dealing with the automatic detection, estimation, and combination of data from multiple sensors. ${ }^{11}$ A key characteristic of the technique is a reliance on multilevel processing and autonomous operation. Sensor fusion methods are particularly well suited to the tasks of state determination and target detection.

For the present study, several different sensor fusion methods were considered for use in generating flow control feedback. After evaluating the advantages and disadvantages of each method, it was determined that a "sensor-level" fusion approach, shown in the block diagram of Figure 3, would be employed. ${ }^{12}$ In this form of fusion, each sensor's output signal is individually conditioned and preprocessed by a series of separate but identical and relatively simple hardware modules. This preprocessing results in the generation of a set of greatly reduced data that is then streamed in real time to a central fusion module. The fusion module classifies the streamed data to produce the final desired information. The main benefits of this architecture are a reduced burden for the fusion module and the use of lower bandwidth data channels for each sensor - a direct result of the preconditioning and processing of the sensor outputs. The architecture is scalable since additional sensors require only duplication of the preprocessing hardware with little or no expansion of the central fusion module.

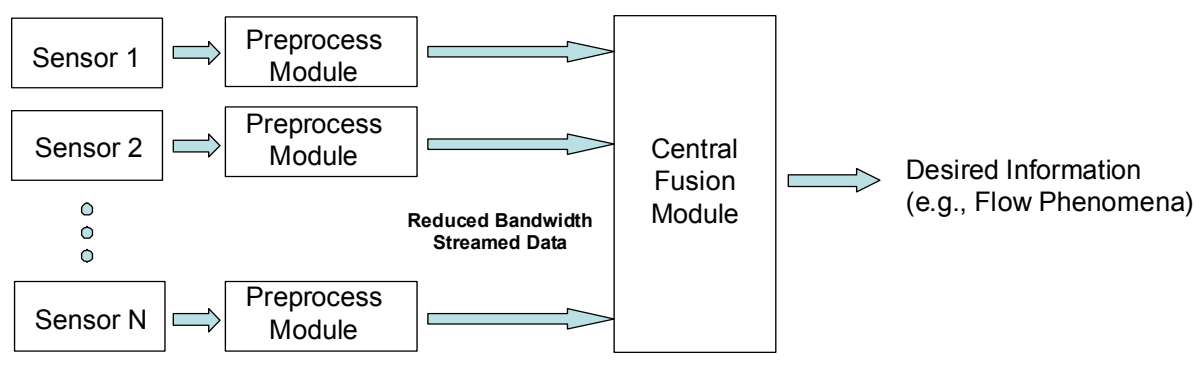

Figure 3. Sensor-Level Fusion.

Incorporation of the sensor-level fusion method shown in Fig. 3 into a flow or noise control system is straightforward. The result is the modified closed loop control system shown in Figure 4. The overall structure of the system is unchanged, the only difference being the addition of the fusion processor between the sensor array and the controller. The remainder of this paper deals with the shaded box shown in Fig. 4 . 


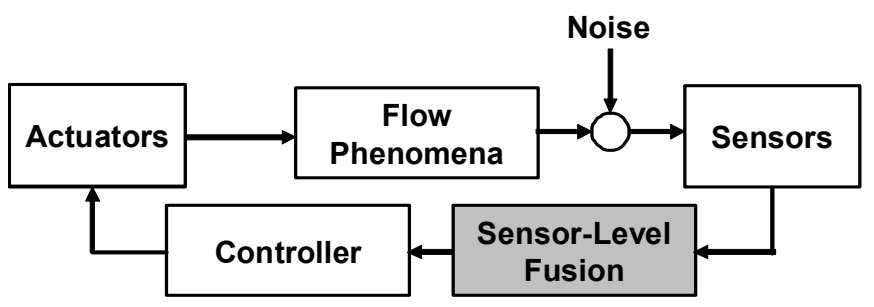

Figure 4. Incorporation of Sensor-Level Fusion into Closed-Loop Control System of Fig. 1.

\section{Prototype Sensor-Level Fusion Processor}

To demonstrate the value of applying the design methods discussed in Section II to flow and noise control, a prototype sensor-level fusion processor was constructed for this study and designed to generate real-time flow state information. Concurrent with the design of the processor was the need to choose a practical flow to investigate as well as a sensor array to interrogate the flow. It is noted that the purpose of the study was not to develop flow control methodologies, but rather to take advantage of a simple flow control concept to facilitate testing of the processor. Criteria for selection of a candidate flow included the ability to generate and manipulate the flow and the ability to provide a relatively non-ambiguous macro flow state at any instant of time. Another requirement was that the flow be of interest to flow and noise control researchers. After considering several candidate phenomena, a simple, low Reynolds number, two-dimensional separated flow, as shown in Figure 5, was selected. There are several benefits to choosing this type of flow phenomenon for testing the processor. In particular, the physics of the surface pressure signatures beneath such flows are described extensively in the literature. Cherry et al., ${ }^{13}$ Farabee et al., ${ }^{14}$ and Hudy, ${ }^{15}$ have obtained measurements of fluctuating wall pressures downstream of forward-facing and backward-facing steps. Their results revealed that the surface pressure fluctuations rise rapidly with increasing streamwise distance from the step and reach a maximum near the reattachment point, as shown in Figure 6.

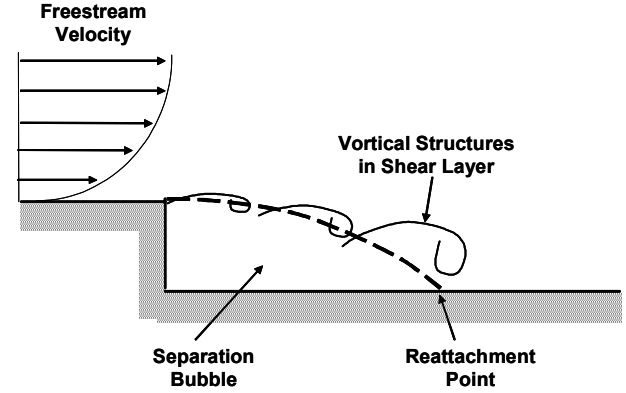

Figure 5. Backward-Facing Step Separated / Reattached Flow.

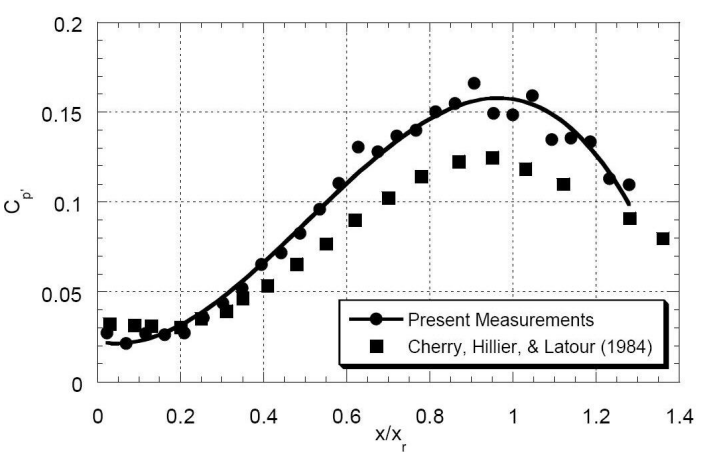

Figure 6. RMS Pressure Distribution Downstream of Back Step in Fig. 5. (from Ref. 15)

The RMS pressure distribution characteristic shown in Fig. 6 can be exploited to track the extent of the separation bubble. Using this knowledge, it was decided to construct a prototype sensor-level fusion processor capable of determining in real-time the two-dimensional flow reattachment point and to show that this information can be used as potential feedback to a flow or noise controller. 


\section{A. Processor Design}

Input to the processor consists of an ensemble of voltages generated by an array of surface-mounted pressure sensors located under the separation bubble shown in Fig. 5. Given this input, the processor must perform the following functions to deduce the desired flow reattachment point from the measurements:

1. The real-time root-mean-square (RMS) pressure is computed at each sensor location

2. The RMS pressures are streamed to a fusion module for comparison and determination of the sensor closest to the reattachment point

3. A simple feedback signal is generated containing the reattachment point information

Figure 7 shows the major components of the architecture required to perform these functions, using the sensor-level fusion technique shown in Fig. 3. The sensor preprocessing section of the design consists of a series of RMS to DC voltage converters, one for each sensor. The outputs of these converters are streamed in parallel to the fusion module shown inside the dotted box in Fig. 7. The basic function of the fusion module is to act as a voting circuit. This voting circuit determines which sensor's RMS level is highest and then feeds that information to a digital-to-analog converter. The converter generates a DC output voltage proportional to the location of the reattachment point. Details follow regarding the individual circuits constructed to perform the core functions shown in Fig. 7.

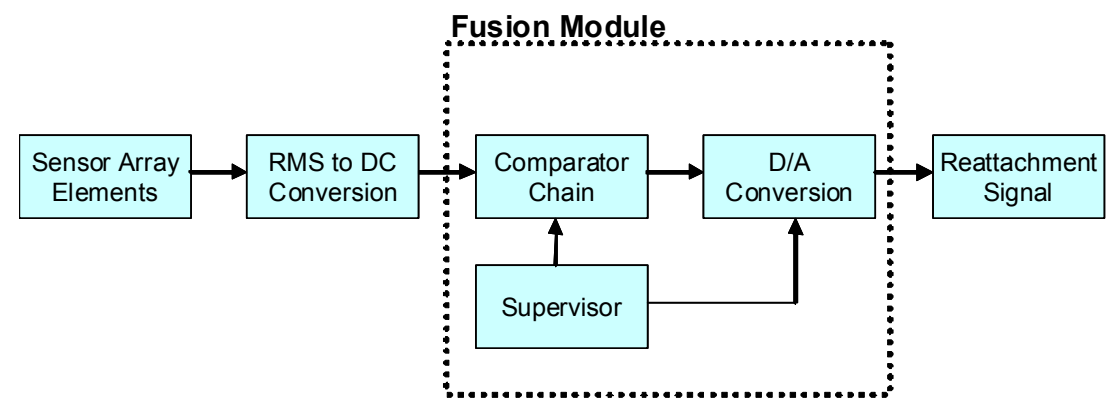

Figure 7. Prototype Processor Architecture.

\section{B. Circuit Specifics}

RMS to DC Conversion: Figure 8 shows a schematic for a simple rapid-response, true-RMS conversion circuit constructed as the preprocessor to the fusion module. The mathematical function computed by the circuit is

$$
V_{R M S}=\sqrt{\frac{1}{T} \int_{0}^{T}\left[V(t)^{2}\right] d t} .
$$

where $V(t)$ is the input voltage, $T$ is the integration time, and $V_{R M S}$ is the RMS value of the input voltage represented as an output DC voltage. The circuit was built around a commercially-available Analog Devices AD736 RMS-to-DC converter and was designed to provide an accurate RMS reading regardless of the type of input waveform being measured. ${ }^{*}$ The preprocessor contained an identical version of the circuit in Fig. 8 for each sensor in the array. Signals from the sensors were AC-coupled to the converter and passed through a resistor divider to scale the input voltages to a level compatible with the input signal range of the AD736. A pair of diodes attached to the converter's voltage rails protected it from signals exceeding

\footnotetext{
* Specific manufacturer's names are explicitly mentioned only to accurately describe the test hardware. The use of manufacturer's names does not imply an endorsement by the U.S. Government nor does it imply that the specified equipment is the best available.
} 
the designed input range. The 1 and $10 \mu \mathrm{F}$ capacitors were chosen to provide a low frequency cutoff of approximately $250 \mathrm{~Hz}$ and maintain the settling time (within one percent of the actual RMS reading) to less than 120 milliseconds. The settling time was chosen to be as short as possible to allow the circuit to operate at greater speed and thus be applicable to higher speed flows.

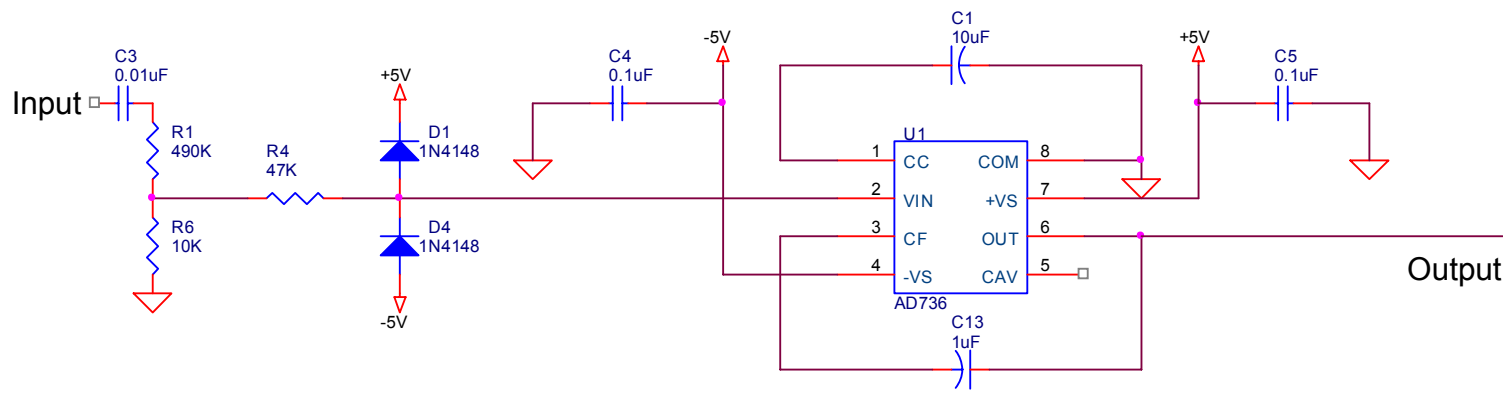

Figure 8. RMS to DC Conversion Circuit.

Comparator Chain: The heart of the fusion module in the dotted box of Fig. 7 was a chain of high-speed LM306 differential voltage comparators, shown in block diagram form in Figure 9. Independent comparators were used for each sensor in the array. The comparator chain acted as a round-robin voting block, identifying in real-time the sensor having the highest RMS voltage level. This operation was performed by applying the RMS-derived DC voltage for each sensor directly to one of the inputs on a corresponding comparator. The DC voltages were also applied to the input of an analog multiplexer that forwarded in turn the voltage produced by each sensor to the other side of each comparator. The multiplexers were controlled via an address line generator and master clock that acted as the supervisor for the circuit. Capture of the comparator outputs was performed using a simple AND gate chain and a 74LS373 octal latch which continually captured and latched the digital address of the sensor exhibiting the maximum RMS voltage. The output of the latch was forwarded to a conversion circuit, described subsequently, for final generation of the representative feedback signal.

While the described comparator chain might at first seem to be an unusual way to implement a fusionbased voting block, the circuit had the advantage of being very simple and fast. It was also scalable via the straightforward addition of comparators and multiple multiplexers, and required no external computer hardware. Note that the circuit was partially simplified by implementing the address generator in a Field-Programmable Gate Array (FPGA). Further simplification of the circuit could be performed by implementing the digital "glue" portions of the circuit (e.g., the AND gate chain) in additional FPGA blocks. The rapid advance in the development of microcontrollers and the availability of economical "systems on a chip" guarantee that future generations of this architecture can be constructed with decreased component count versus the prototype built for this study.

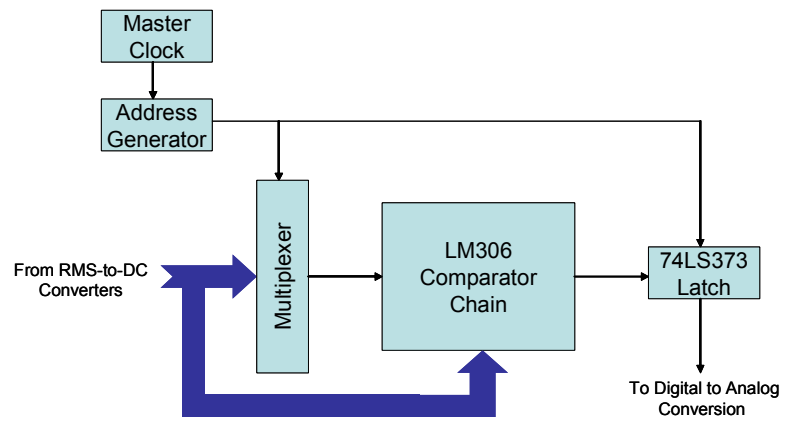

Figure 9. Comparator Chain Block Diagram. 
Digital-to-Analog (D/A) Conversion: Figure 10 shows a partial schematic for the circuit performing the conversion of the digital address of the sensor with the maximum RMS level to a feedback control signal. The feedback signal was defined as a simple DC voltage proportional to the sensor address, and was produced using an 8-bit DAC0808 D/A integrated circuit coupled with an LF351 operational amplifier. Only the five most significant bits of the address were used in the current implementation of the circuit. The output voltage after conversion is represented by the equation

$$
\text { Vout }=\operatorname{Vref}\left[\frac{A 1}{2}+\frac{A 2}{4}+\frac{A 3}{8}+\frac{A 4}{16}+\frac{A 5}{32}\right]
$$

where the $V_{\text {ref }}$ reference voltage is 10 volts.

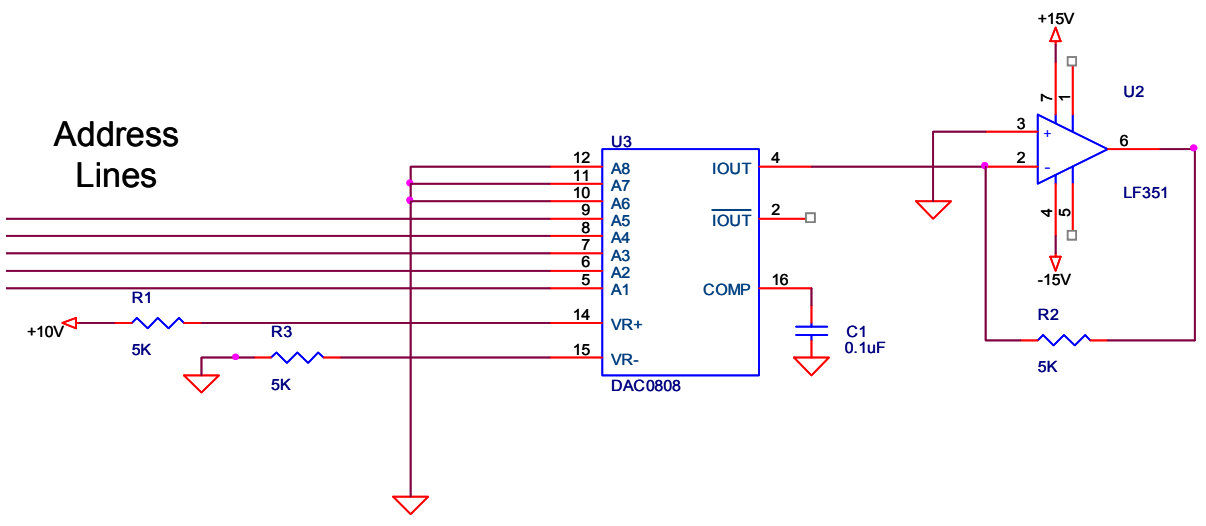

Figure 10. Digital-to-Analog Conversion.

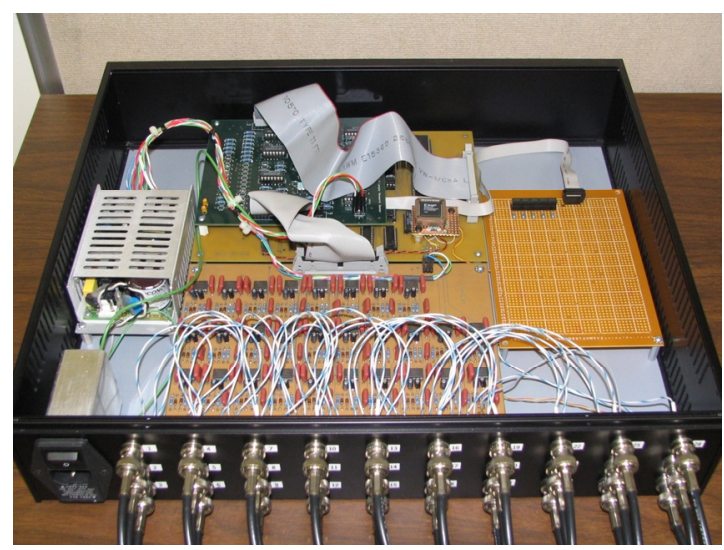

Figure 11. Completed Prototype Processor.

\section{Processor Testing and Validation}

Testing and validation of the completed processor, shown in Figure 11, were conducted using data obtained from a low-speed wind tunnel test. The wind tunnel test was performed on a separated flow model previously used during an Integrated Instrumentation and Testing Systems (IITS) Unified Instrumentation Experiment. ${ }^{16}$ Note that wind tunnel testing of the processor was conducted purely as an instrumentation 
development exercise to assess the performance of the hardware under realistic conditions. The purpose of the testing was not to study flow physics. Validation of the processor was achieved by comparing the flow reattachment location produced by the processor with similar information derived from direct analysis of acquired sensor data and from Particle Image Velocimetry (PIV) mean velocity fields obtained in the vicinity of the upper surface of the model. Both hybrid and direct testing of the processor were performed. Hybrid testing of the processor consisted of feeding model pressure sensor data into the front end of the architecture shown in Fig. 7 while the fusion module circuit was simulated within Matlab. Direct testing of the processor consisted of feeding pressure data into the complete architecture of Fig. 7.

\section{A. Test Description}

Test Apparatus: Testing was conducted in the LaRC Subsonic Basic Research Tunnel (SBRT), shown in Figure 12. The SBRT is a subsonic, open-return wind tunnel with a 0.56-x 0.81-x 1.86-meter test section, that can be operated at atmospheric pressure and is capable of producing a maximum freestream velocity of approximately $50 \mathrm{~m} / \mathrm{sec}$. The test section is equally divided into upstream and downstream bays with removable sidewalls to facilitate model installation and test section access.

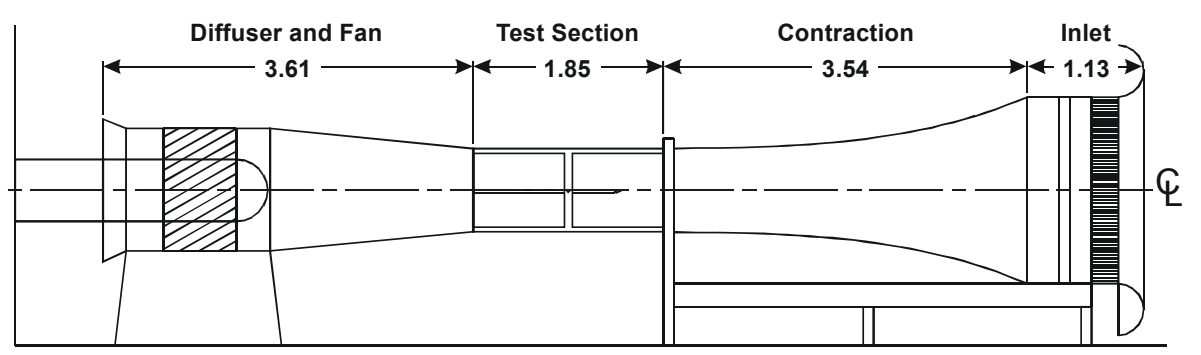

Figure 12. NASA Langley Subsonic Basic Research Tunnel. (Dimensions in meters)

The model employed for this study was the same one used in the IITS investigation and consisted of a flat plate with a hinged leading edge, shown in Figure 13. This configuration was chosen specifically for instrument development purposes as opposed to a model with a classical aerodynamic shape. Careful consideration was given to designing a model that generated structural and flow characteristics that could be resolved by a pressure sensor array. The flat plate leading edge angle (LEA) was adjustable from $-12^{\circ}$ to $+12^{\circ}$. This enabled a flow separation bubble to be generated at positive inclination angles. A clamping block was used to hold the leading edge rigidly in place for static LEA testing. A DC motor was attached to a bracket connected to the model leading edge to facilitate oscillatory LEA testing.

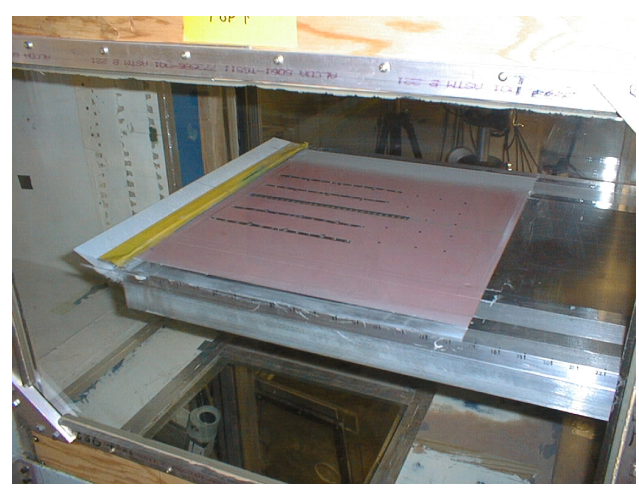

Figure 13. Separated Flow Test Model. 
An instrumented plate (I-plate) containing an array of pressure sensors was installed on the upper surface of the model near the leading edge. This enabled the acquisition of unsteady pressure measurements in the vicinity of flow separation and reattachment. The I-plate used for this study was previously employed to characterize the separated flow downstream of a backward step as described in Ref. 15. The plate was populated with 80 flush-mounted electret microphones acting as hydrodynamic pressure sensors. The microphones were arranged in five streamwise rows with 28 sensors situated along the centerline of the plate and two rows each containing 13 sensors located on either side of the centerline. Figure 14 illustrates the location of these sensors. For the present study, only the outputs from the centerline row of 28 sensors were applied to inputs on the prototype processor. Using Eq. (2), the output voltage mappings shown in Table 1 were defined for each sensor on the I-plate centerline, making the output voltage of the processor proportional to the location of the sensor closest to reattachment.

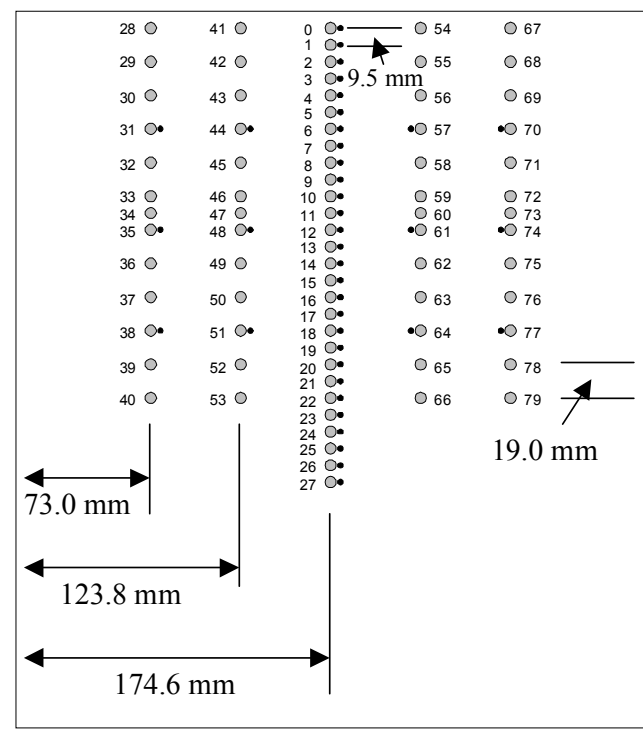

\section{Figure 14. Instrumented Sensor Plate. Large Circles $=$ Microphones Small Dots $=$ Static Pressure Taps}

Data Acquisition: Two distinct modes of data acquisition were performed to acquire information from the I-plate sensor array. The traditional acquisition system shown in Fig. 2 was employed to digitize the sensor voltage time histories for analysis and comparison with the processor output. The acquisition system utilized a 64-channel anti-alias filter bank coupled with a 16-bit multiplexed data acquisition card residing in a PC class computer. Each sensor was sampled at $6 \mathrm{kHz}$, well above the estimated spectral bandwidth of $1 \mathrm{kHz}$ anticipated in the separated flow field generated above the I-plate. A 25-second time history record was typically collected during this mode of acquisition.

The second mode consisted of applying the output voltages of the 28 I-plate centerline sensors to an analog data recorder. The analog recorder was capable of simultaneous recording a maximum of 32 channels of analog data in real time to research-quality VHS tapes. A typical acquisition cycle consisted of recording 25 seconds of data for playback through the processor. This mode of acquisition was ideal for allowing circuit modifications and adjustments to be tested without the need for repeating tunnel runs, and ensured a consistent data input to the processor. Captured signals were played through the processor on demand using the setup shown in Figure 15.

Test Matrix: The test matrix included both static and dynamic LEA settings for a range of freestream velocities. The relevant model and tunnel conditions are summarized in Table 2. For static testing, data ensembles were collected for seven static LEA's at freestream velocities ranging from 15 to $40 \mathrm{~m} / \mathrm{sec}$. 
Dynamic LEA test data were acquired over an LEA range of -12 to +12 degrees with the leading edge oscillating at a 1-Hertz rate. Only data acquired at a freestream velocity of $40 \mathrm{~m} / \mathrm{sec}$ is presented here.

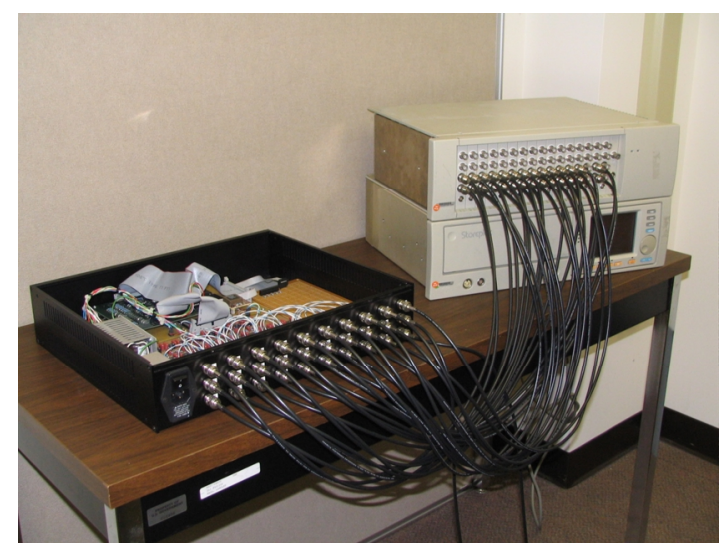

Figure 15. Processor with Analog Data Recorder.

Table 1. Processor Sensor to Voltage Mapping

\begin{tabular}{|c|c|c|c|}
\hline Sensor & Output Voltage & Sensor & Output Voltage \\
\hline 0 & 0.00 & 14 & 4.38 \\
\hline 1 & 0.31 & 15 & 4.69 \\
\hline 2 & 0.63 & 16 & 5.00 \\
\hline 3 & 0.94 & 17 & 5.31 \\
\hline 4 & 1.25 & 18 & 5.63 \\
\hline 5 & 1.56 & 19 & 5.94 \\
\hline 6 & 1.88 & 20 & 6.25 \\
\hline 7 & 2.19 & 21 & 6.56 \\
\hline 8 & 2.50 & 22 & 6.88 \\
\hline 9 & 2.81 & 23 & 7.19 \\
\hline 10 & 3.13 & 24 & 7.50 \\
\hline 11 & 3.44 & 25 & 7.81 \\
\hline 12 & 3.75 & 26 & 8.13 \\
\hline 13 & 4.06 & 27 & 8.44 \\
\hline
\end{tabular}

Table 2. Wind Tunnel / Model Conditions

\begin{tabular}{|l|l|}
\hline Freestream Velocity Range, $U_{\infty}$ & 15 to $40 \mathrm{~m} / \mathrm{sec}$ \\
\hline Static Leading Edge Angles, $\alpha$ & $0,2,4,6,8,10,12 \mathrm{deg}$ \\
\hline Dynamic Leading Edge Angle Range & -12 to $+12 \mathrm{deg}$ \\
\hline Dynamic Leading Edge Oscillation Freq, $f_{\text {OSC }}$ & $1 \mathrm{Hertz}$ \\
\hline
\end{tabular}

\section{B. Representative Results}

Static Testing: Figure 16 depicts a typical 3-second snapshot of the output signal generated by the fusion processor when sampling the flow state for a model LEA of eight degrees and a freestream velocity of $40 \mathrm{~m} / \mathrm{sec}$. Input to the processor was via the analog recorder, and the processor was configured to sample the sensor data at a $60-\mathrm{Hz}$ rate. Both the processor output voltage and the corresponding reattachment distance measured from the leading edge of the I-plate are shown in the figure. Recall that the latching circuit in Fig. 9 drives the output of the D/A converter in the discrete voltage steps listed in Table 1. This behavior appears as the quantized levels seen in the voltage time history shown in Fig. 16. The 
quantization seen in Fig. 16 is due to the spatial geometry of the sensor array and not due to voltage or temporal quantizations. Note that the processor's quantization error is directly related to the packing density of the sensors in the array. Decreasing the sensor spacing on the I-plate would thus decrease the observed quantization error.

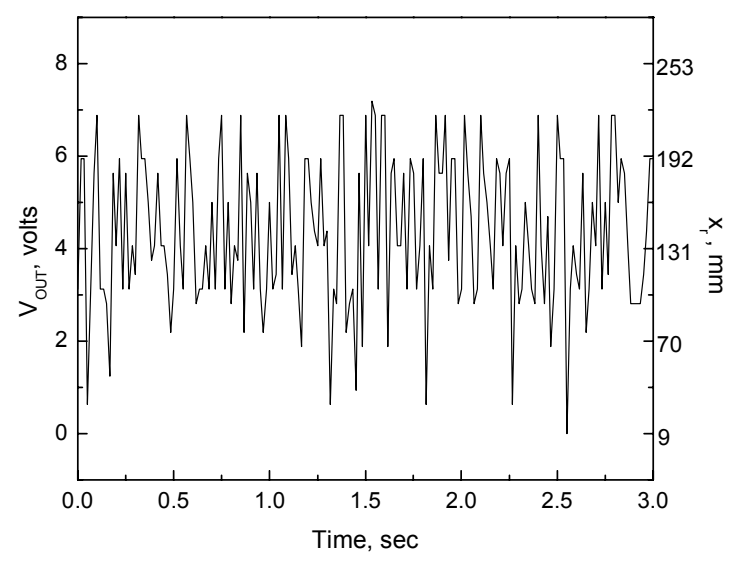

\section{Figure 16. Typical Processor Output Signal Time History. LEA $=\mathbf{8}^{\circ}, U_{\infty}=\mathbf{4 0} \mathrm{m} / \mathrm{sec}$.}

Figure 17 shows the mean reattachment distance as deduced by the processor and the extent of change in that distance for a range of LEA's at a freestream velocity of $40 \mathrm{~m} / \mathrm{sec}$. Ensembles of 25 -second voltage time histories with features similar to that shown in Fig. 16 were used to generate the results shown in Fig. 17. As can be seen in the figure, the reattachment distance is a nonlinear function of the LEA, and the spatial extent of movement of the reattachment point as a function of time increases with increasing LEA. This is shown in Fig. 17 by the bars bracketing each symbol. These are not error bars in the traditional sense, but rather show the increase in the turbulent fluctuations in the separation bubble as the LEA is increased.

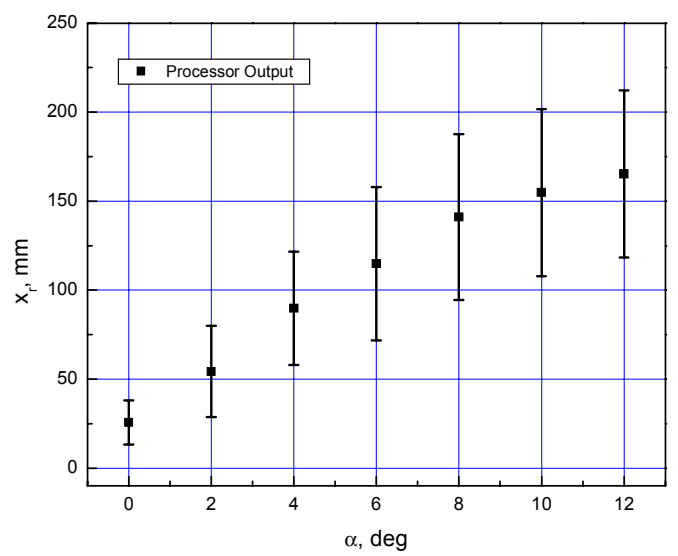

Figure 17. Mean Reattachment Distance Detected by Processor. $U_{\infty}=40 \mathrm{~m} / \mathrm{sec}$.

The data shown in Fig. 17 were validated via direct analysis of ensembles of sensor time history data acquired using the traditional acquisition system shown in Fig. 2. The analysis consisted of computing the 
RMS voltage fluctuation measured by each sensor. This was done by converting the recorded raw counts for each sensor into engineering units (volts). The fluctuations were then computed via

$$
V_{i, R M S}=\sqrt{\frac{\sum_{k=1, n}\left(v_{k}-\bar{v}\right)^{2}}{n-1}}
$$

where $v_{k}$ is the $\mathrm{k}^{\text {th }}$ voltage sample from an $\mathrm{n}$-sized ensemble acquired for sensor $i$.

The performance of the processor output was further validated by comparing resultant data sets with a series of PIV mean velocity fields. These velocity fields were acquired in the vicinity of the upper surface of the model and used to determine the flow reattachment location as a function of LEA and freestream velocity. Details regarding the acquisition and basic processing of the PIV data are given in Ref. 16. Figure 18 depicts a series of six streamline plots as derived from their corresponding mean velocity fields. The streamlines are shown for static LEA's of 2, 4, 6, 8, 10, and 12 degrees, acquired at a freestream velocity of $40 \mathrm{~m} / \mathrm{sec}$. The horizontal axis in each plot represents the distance from the leading edge of the I-plate. The flow reattachment can be clearly seen in these plots as that point where the flow impinges normal to the model surface. Upstream of this location the flow direction is reversed with respect to the freestream velocity direction. To accurately determine the reattachment location for comparison with the processor output, sets of $U$ (streamwise) velocity component data were extracted along the surface of the model for each mean velocity field. The streamwise velocity zero crossing point was then identified in each set of extracted data using a standard linear regression model. The crossing point corresponds exactly to the flow reattachment location. Figure 19 shows an example of one of the zero crossing functions for an LEA of six degrees and freestream velocity of $40 \mathrm{~m} / \mathrm{sec}$.

Figure 20 shows the reattachment location information derived from analysis of the sensor time history data and PIV mean velocity fields, superimposed on the processor data shown in Fig. 17. There is excellent agreement between the processor output and the direct analysis of the time history data. This is not surprising because the basic underlying principle of the processor is the real-time determination of RMS voltages measured by the sensors. Note that there is a consistent positive bias in the reattachment location as determined from the PIV analysis. This bias grows larger as the LEA is increased. It is documented that peak RMS pressure fluctuations occur slightly upstream of the actual reattachment location in backwardfacing step flows. As reported by Heenan et al., ${ }^{17}$ and further verified by Hudy in Ref. 15 and shown in Fig. 6, maximum RMS pressure fluctuations typically occur one-step height upstream of reattachment. Given the geometry of the hinged leading edge model employed for the current study, the PIV reattachment location bias shown in Fig. 20 is proportional to the step height for the majority of LEA's employed in the study. In an actual separated flow control application using the processor, this bias error could be nulled in the processor output to improve the accuracy of the flow state determination; however, this was not performed for the current study.

The static testing verifies that the prototype processor is indeed capable of producing reasonably accurate estimates of the separation bubble extent as a function of static leading edge angle. Similar results to those shown in Figs. 17 and 20 were obtained for the other freestream velocities listed in Table 2. 

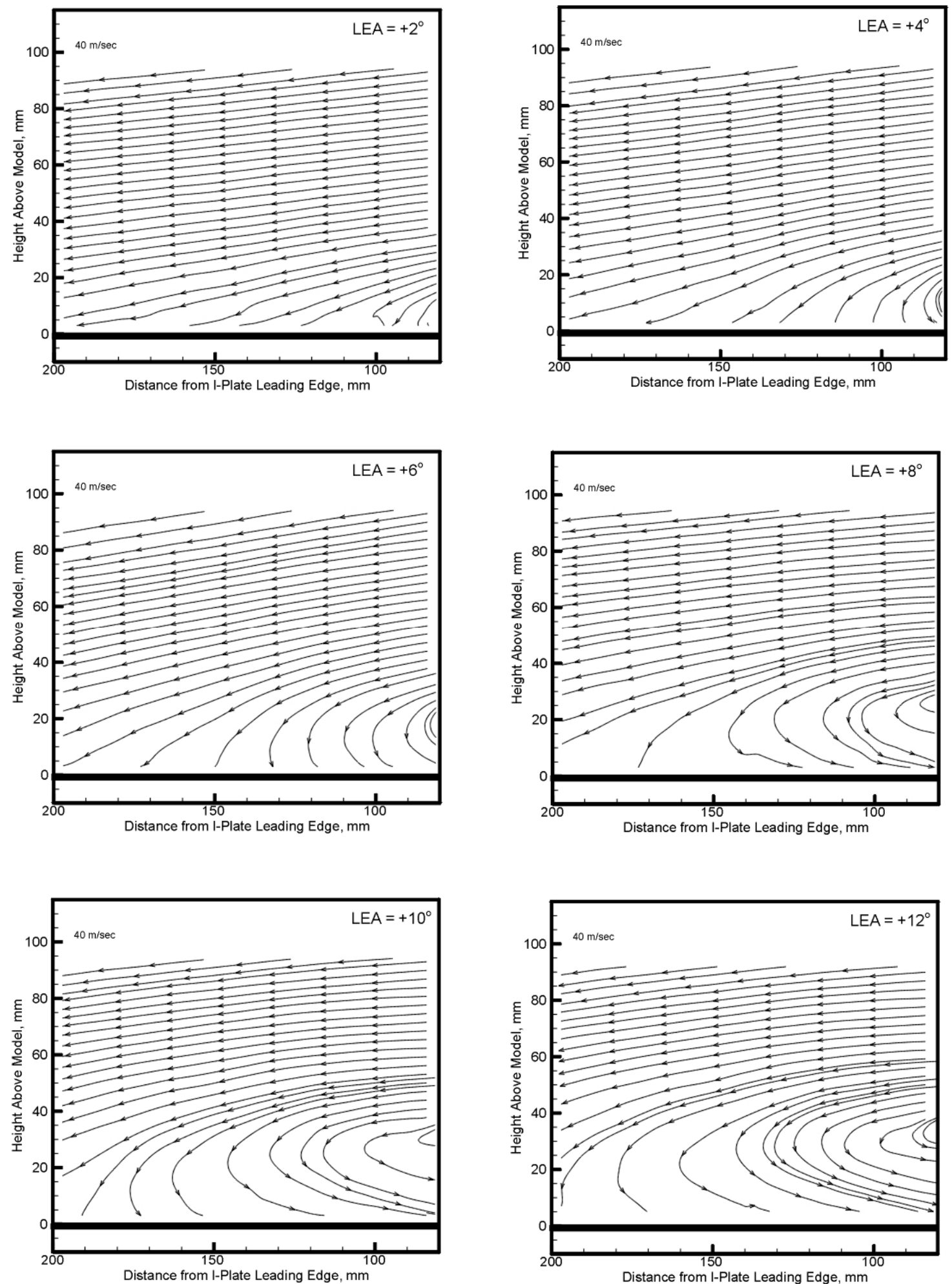

Figure 18. PIV Velocity Streamlines for $\operatorname{LEA}=2,4,6,8,10$, and $12^{\circ}$. Freestream velocity $=\mathbf{4 0} \mathrm{m} / \mathrm{sec}$.

American Institute of Aeronautics and Astronautics 


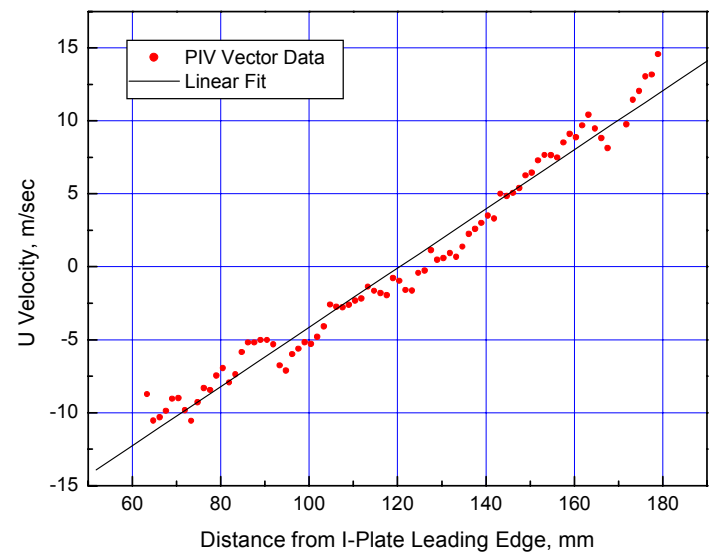

Figure 19. PIV Zero-Crossing Function. $\mathrm{LEA}=6^{0}, U_{\infty}=40 \mathrm{~m} / \mathrm{sec}$.

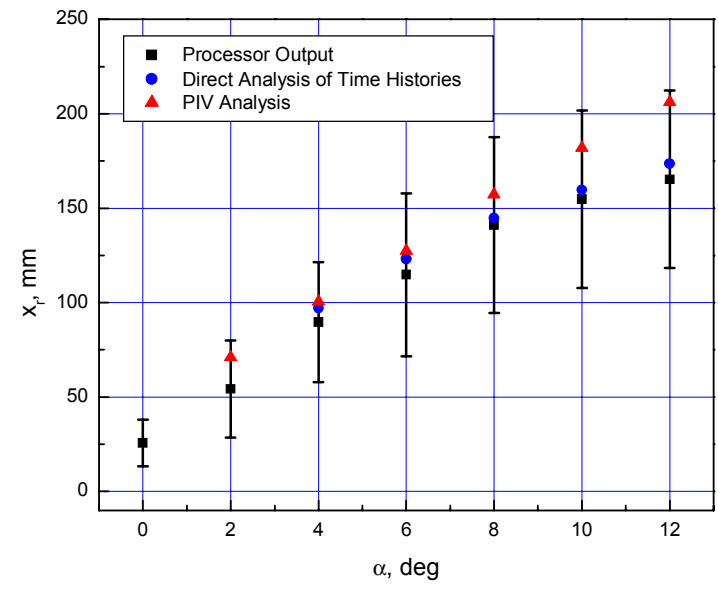

Figure 20. Comparison of Reattachment Distance Measures. $U_{\infty}=40 \mathrm{~m} / \mathrm{sec}$.

Dynamic Testing: Figure 21 depicts the time history output of the processor when sampling the separated flow while the leading edge of the model was dynamically oscillated. The leading edge oscillation frequency was $1 \mathrm{Hertz}$ and the freestream velocity was $40 \mathrm{~m} / \mathrm{sec}$. The processor was configured to sample the sensors at a 64-Hz rate. Two seconds of real-time data are shown in Fig. 21, representing two complete oscillations of the model leading edge. The reattachment distance in Fig. 21 has been normalized by the maximum $x_{r}$. Sections of time where the LEA is negative can be clearly identified in the figure from regions where no output voltage is produced by the processor. This is the result of little or no flow separation occurring on the upper surface of the model. Correspondingly, no sensor measures a dominant RMS pressure. When the LEA crosses zero and moves toward +12 degrees, the separation bubble produced on the upper surface of the model increases in size and the reattachment point moves downstream. As a result, the maximum RMS pressure travels down the array of sensors, producing a processor output voltage proportional to the index of the sensor registering the highest RMS pressure and thus being closest to reattachment. The time history shown in Fig. 21 is the type of information that would be relayed to a flow or noise controller like that shown in Fig. 3. Filtering of the processor output could be 
performed to smooth the signal and improve the robustness of the system in the handling of reattachment point transient fluctuations like those shown in Fig. 21. Figure 22 illustrates an example of such filtering using a 5-point FFT smoothing filter.

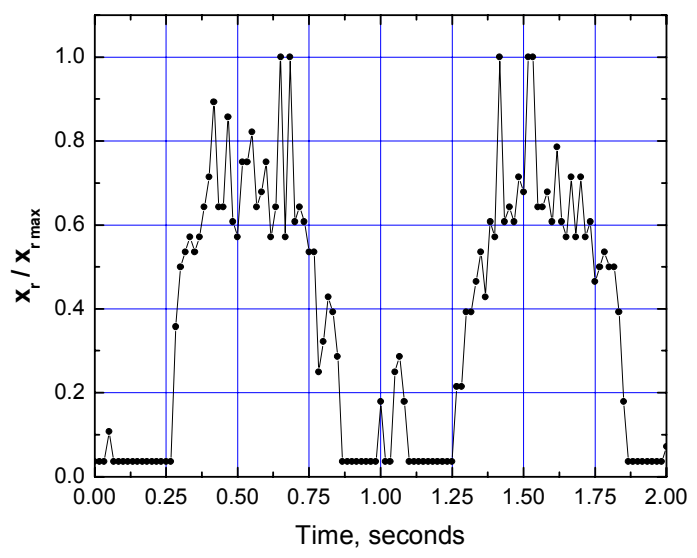

Figure 21. Dynamic Processor Output. LEA Range of -12 to +12 Degrees, $f_{\text {osc }}=1.0 \mathrm{~Hz}, V_{\infty}=40 \mathrm{~m} / \mathrm{sec}$.

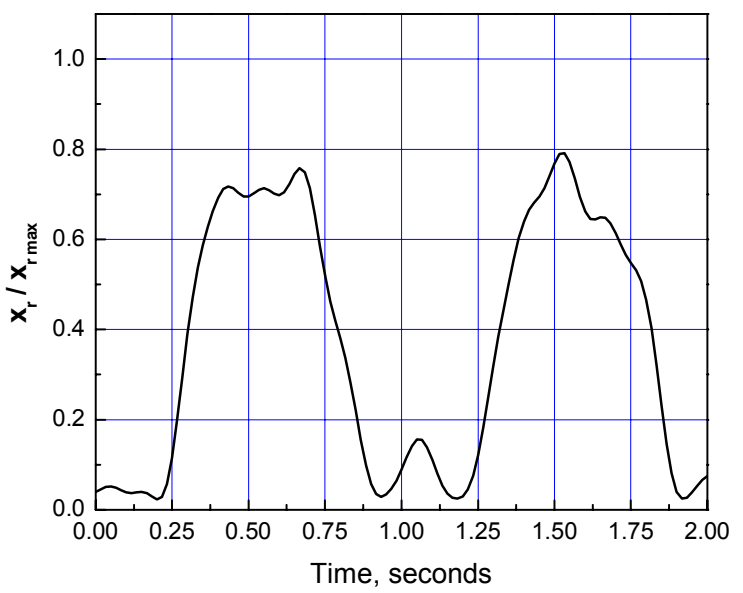

Figure 22. Output of Fig. 21 Smoothed Using FFT Filter.

\section{Summary}

Multi-channel sensor fusion represents a powerful technique to simply and efficiently extract information from complex phenomena. While the technique has traditionally been used for military target tracking and situational awareness, the current study has demonstrated that sensor fusion can be applied equally well to aerodynamic applications. In particular, the "sensor-level" method of fusion is particularly well suited to the task of state determination. As such, the technique can be applied in the reduction of sensor array data as part of a flow or noise control feedback system. To demonstrate the potential of these new types of architectures, a prototype sensor-level fusion processor was successfully designed and used to detect in real-time the flow reattachment location generated by a simple wind tunnel model. This demonstration 
clearly illustrates the feasibility of using these new architectures for enhancing flow control sensor array analysis and feedback signal generation.

\section{Acknowledgements}

The authors gratefully acknowledge James K. Adams (Langley Electronic Systems Branch), Scott M. Bartram, (Langley Advanced Sensing and Optical Measurement Branch), and Angelo A. Cavone (ATK Space Division), for their helpful advice and assistance with the design, fabrication, and testing of the sensor fusion processor. The authors also acknowledge the support of Dr. Laura M. Hudy (General Electric Research) and Dr. Ahmed M. Naguib (Michigan State University) for their helpful advice regarding the wind tunnel testing and validation of the processor.

\section{References}

1. Collis, S. S., Joslin, R. D., Seifert, A., and Theofilis, V, "Issues in Active Flow Control: Theory, Control, Simulation, and Experiment", Progress in Aerospace Sciences, Vol. 40, pp. 237-289, 2004.

2. Kuo, S. M., and Morgan, D. R., "Active Noise Control: A Tutorial Review", Proceedings of the IEEE, Vol. 87, No. 6, pp. 943 - 973, 1999.

3. Thomas, R. H., Choudhari, M. M., and Joslin, R. D., "Flow and Noise Control Review and Assessment of Future Directions," NASA Technical Memorandum 2002-211631, 2002.

4. Joslin, R. D., Horta, L. G., and Chen, F. J., "Transitioning Active Flow Control to Applications", AIAA Paper 1999-3575, 30 $0^{\text {th }}$ AIAA Fluid Dynamics Conference, Norfolk, VA, 1999.

5. Schaeffler, N. W., Hepner, T. E., Jones, G. S., and Kegerise, M. A., "Overview of Active Flow Control Actuator Development at NASA Langley Research Center," AIAA Paper 2002-3159, ${ }^{\text {st }}$ AIAA Flow Control Conference, St. Louis, MO, 2002.

6. Cattafesta, L. N., Garg, S., and Shukla, D., "Development of Piezoelectric Actuators for Active Flow Control," AIAA Journal, Vol. 39, No. 8, pp. 1562-1568, 2001.

7. Wright, K. D., et al., "Measurement Technology for Use in Active Flow Control," AIAA Paper 2002$2705,22^{\text {nd }}$ AIAA Aerodynamic Measurement Technology and Ground Testing Conference, St. Louis, MO, 2002.

8. Tenney, R. R., and Sandell, N. R., "Detection with Distributed Sensors," IEEE Transactions on Aerospace and Electronic Systems, Vol. AES-17, No. 4, pp. 501-509, 1981.

9. Veeravalli, V. V., "Decentralized Sequential Detection with a Fusion Center Performing the Sequential Test," IEEE Transactions on Information Theory, Vol. 39, No. 2, pp. 433-442, 1993.

10. Willett, P., Alford, M., and Vannicola, V., "The Case for Like-Sensor Pre-detection Fusion," IEEE Transactions on Aerospace and Electronic Systems, Vol. 304, No. 4, pp. 986-1000, 1994.

11. Hall, D. L., and Llinas, J. (ed.), "Handbook of Multisensor Data Fusion," CRC Press, 2001.

12. Klein, L. A., "Sensor and Data Fusion Concepts and Applications," SPIE Optical Engineering Press, 1993.

13. Cherry, N. J., Hillier, R., and Latour, M., "Unsteady Measurements in a Separated and Reattaching Flow,” Journal of Fluid Mechanics, Vol. 144, pp. 13-46, 1984.

14. Farabee, T. M., and Casarella, M. J., "Measurements of Fluctuating Wall Pressure for Separated / Reattached Boundary Layer Flows," Journal of Vibration, Acoustics, Stress, and Reliability in Design, Vol. 108, pp. 301-307, 1986.

15. Hudy, L. M., "Simultaneous Wall-Pressure Array and PIV Measurements in a Separating / Reattaching Flow Region," Master's Thesis, Michigan State University, 2001.

16. Fleming, G. A. (ed.), "Unified Instrumentation: Examining the Simultaneous Application of Advanced Measurement Techniques for Increased Wind Tunnel Testing Capability," AIAA Paper 2002-3244, $22^{\text {nd }}$ AIAA Aerodynamic Measurement Technology and Ground Testing Conference, St. Louis, MO, 2002.

17. Heenan, A. F., and Morrison, J. F., "Passive Control of Pressure Fluctuations Generated by Separated Flows", AIAA Journal, Vol. 36, pp. 1014-1022, 1998. 